\title{
Dynamic Rupture Propagation Modeling
}

by Satoshi Ide

doi:10.2204/iodp.sd.s01.05.2007

Resolving earthquake dynamic rupture is essential to understand the physics behind earthquake rupture. This paper first reviews the current status of the slip inversion, and then discusses the scaling of earthquake dynamic rupture.

Slip inversion methods have been developed since the 1980 s to study the heterogeneity of spatio-temporal slip distribution of earthquakes. Several methods have been applied to many earthquakes using various data, such as near-field strong-motion records, far-field broadband records, and geodetic observations including GPS and InSAR. Some attempts have been made to compile a catalog of slip models to study their general features. Increasing the amount of data and communication speed allows even quasi-real time analysis of slip inversion, which gives an impression that these analyses are easy and straightforward. However, each slip model is based on some assumptions that are not always described well in research papers and can be a reason for apparent large discrepancies between models. For example, the models presented for the 1999 Izmit, Turkey earthquake (Mw 7.6) were quite different (Fig. 1) as shown in Ide et al. (2005). Basic characteristics, such as the locations of large slip and even the starting point of the rupture, were quite different. The data seem not to be insufficient; some strong motion stations are located close to the surface trace of the source fault, and some geodetic measurements are available. However, mainly due to uneven distribution of stations and inaccurate timings of seismometers, the data cannot resolve the growth process of earthquake rupture and the assumptions made by each research group to determine the characteristics of their slip model.

We have to use many model-dependent assumptions to obtain slip models. These include data selection and preprocessing, model settings such as fault plane geometry and parameterization, and inversion method including the assumption of probability function of error. Among them, the most uncertain factor is how to calculate synthetic data that we compare with observed data. Usually we assume layered structures to calculate seismic waveforms. It has been demonstrated that a reliable 3-D structure can increase the resolution of slip inversion, but such structure information is practically unavailable in many cases. The method of Empirical Green's functions, which uses the records of small earthquakes as theoretical waves, provides a good approximation of waves in complex structures, but to find a set of good small events is not easy. Computation of seismic waveform is the bottleneck of current slip inversion.

Even now, the number of well resolved earthquakes is limited to about ten. Nevertheless, we find some general features of slip models. Earthquakes generally consist of successive ruptures of subevents, which are sometimes referred to as asperities with vague definition. The pulse-like rupture propagation is now widely accepted. As reported for some earthquakes, such as the 1906 San Francisco and the 2002 Denali, rupture pulses can propagate faster than the S-wave speed, but the average propagation velocity does not exceed it. Well resolved slip models can be used to discuss dynamic properties of earthquake rupture. Fault constitutive relationships and their characteristic parameters, slip weakening distance $D_{c}$ and fracture energy $G_{c}$, have been estimated for some well resolved earthquakes, such as the 1992 Landers, the 1995 Kobe, and the 2000 Tottori. While $D_{c}$ tends to be overestimated, $G_{c}$ is a relatively stable parameter controlled mainly by rupture propagation velocity.

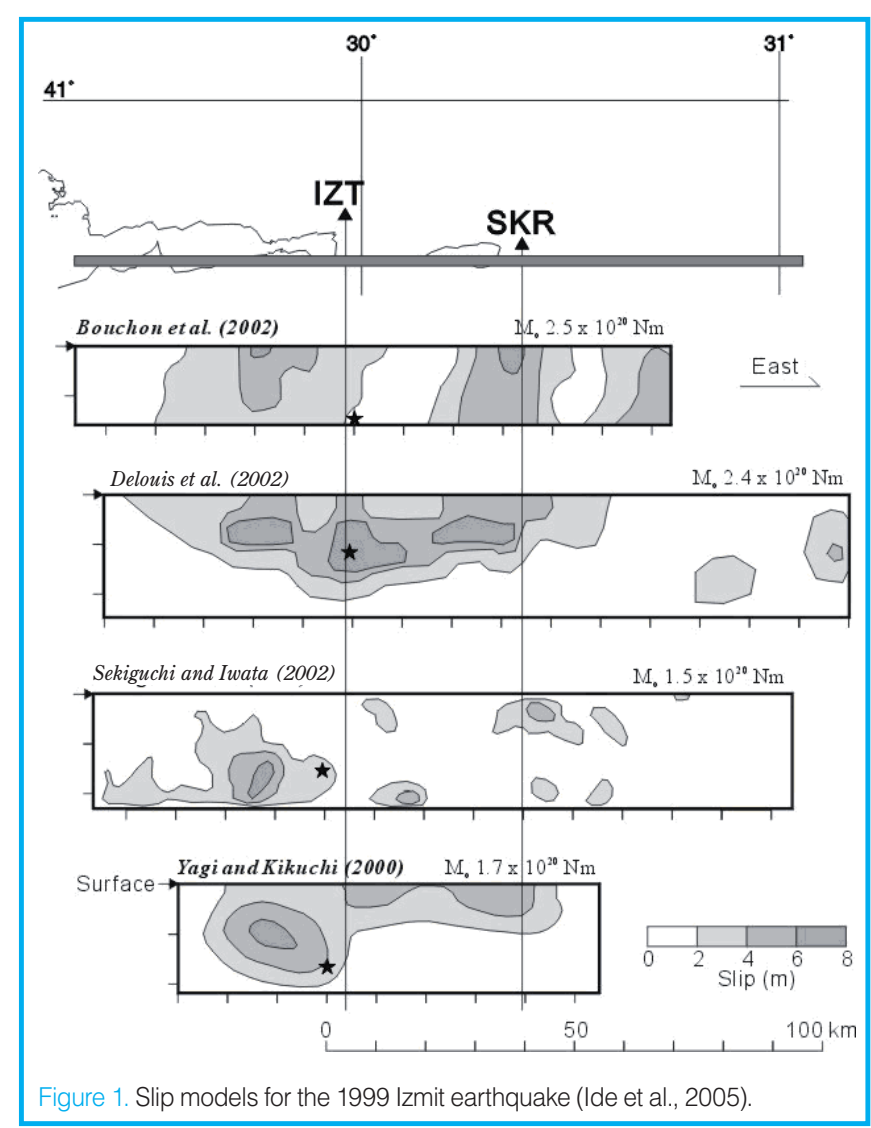


Earthquake faults and slip generally satisfy geometrical similarity; in other words, earthquakes have similar stress drop. If earthquakes are also temporally similar having similar rupture velocities, we expect to observe an almost constant ratio between seismic energy and seismic moment. Although there is still room for discussion whether the ratio is strictly constant, its change is small compared to the wide difference in seismic moment (about 15-fold).

While the scaling for these macroscopic parameters is well documented, the study for the scaling of earthquake complexity has started only recently. For example, Somerville et al. (1999) pointed out that the size of asperity (large slipped areas) scales as the square of the cubic root of seismic moment, suggesting geometrical similarity. Using a large catalog of slip models, Mai and Beroza (2000) also found similar scaling for seismic moment and fault length and width. However, they also showed that the scaling is different for the width of strike slip events, which is an evidence of a break of self-similarity. Sometimes, small earthquakes are assumed to be simple compared to large ones; however, this concept is not so obvious. Moreover, recently estimated slip models for microearthquakes ( Mw 1$)$ in a South Africa gold mine (Yamada et al., 2005) do have a subevent sequence, and characteristic time, length, and slip amount are all smaller by a factor of 1000 compared to those of the Kobe earthquakes. The information about scaling laws and their limitation are quite useful to construct hypothetical earthquake models for strong motion prediction.
Slip models can be used to scale dynamic parameters, too. Fracture energy $G_{c}$ is a relatively reliable parameter calculated for many slip models. The average fracture energy estimated for earthquakes of $\mathrm{M}_{\mathrm{w}} 5$ to 7 is almost proportional to seismic moment, although the examples are limited.

All the previously mentioned studies for earthquake scaling compare small and large earthquakes using different measures that are comparable to the final size of events, but there are other ways to compare. One is the scaling of complexity during rupture growth of one earthquakenamely, comparison between the initial and final stages of one event. Once we recognize this scaling law, we can recognize whether one small rupture is the initial part of a large event or just a small event. Unfortunately, traditional slip inversion cannot solve this problem. Recently, we developed a new method suitable to this problem, which we refer to as the multiscale slip inversion method (Uchide and Ide, 2006). The initial stage of an earthquake can be investigated using high-frequency seismic waves near the onset of signal, while traditional inversion details overall rupture propagation. We can prepare a set of observation equations on these two scales, or with some additional intermediate scales. These observation equations are unified into a multiscale observation equation using a renormalized multiscale slip model. By solving this equation in the same way as traditional slip inversion problem, we obtain a multiscale slip model with changing resolution as the rupture grows.

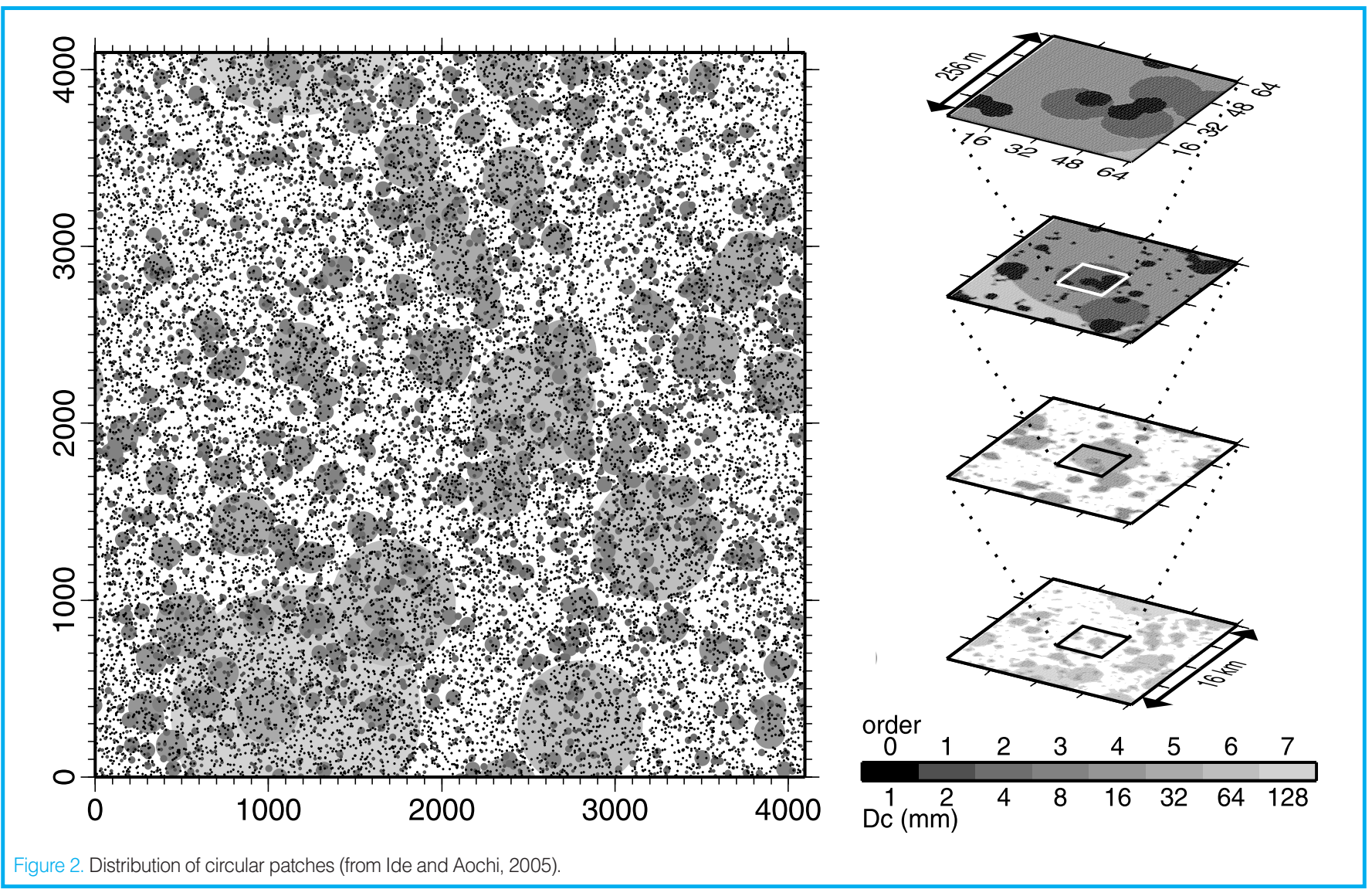


We first applied this method to the mid-Niigata prefecture earthquake in 2004 in Japan. The spatio-temporal slip distribution is obtained as a nested spatio-temporal slip distribution on an assumed fault plane. Unlike ordinary slip models, this model contains a detailed rupture process in the first $1 \mathrm{~s}$. The resolution in the next $1 \mathrm{~s}$ is worse, but the model area increases. Finally, we obtain the full scale rupture process with low resolution, which is similar to the result of traditional slip inversion. We observe several slip pulses in different directions even in the first $1 \mathrm{~s}$. In every scale, slip rate is about $1 \mathrm{~m} \mathrm{~s}^{-1}$, suggesting similarity.

When the earthquake is a self-similar process, how does a rupture propagate? Ide and Aochi (2005) have modeled selfsimilar dynamic ruptures assuming a heterogeneous distribution of circular patches on a planar crack (Fig. 2). Two points of the model are that 1) slip weakening distance $D_{c}$ scales with the size of patch, and 2) the size-number distribution of patches satisfies a power law. Assuming that a rupture starts from one of the smallest patches, we solve the elastodynamic equation using the boundary integral equation method with the renormalization technique. Some ruptures grow into very large events, while most ruptures remain small. The moment rate function of a large event begins with a so-called initial phase which may also contain an initial phase in a smaller scale. Therefore, the rupture process is statistically self-similar. The rupture speed is subshear in average, but it can locally exceed S-wave speed, which is consistent with recent observations and slip models.

Whether a self-similar dynamic rupture growth is a good approximation of an earthquake remains to be proven. The answer to this question is important, not only because it is fundamental for the physics behind earthquakes, but also because it can be the basis of various practical usages of small earthquakes, such as assessment of earthquake predictability and predication of strong motion. Slip inversion is a useful tool to assist in resolving this question, and we have to overcome many problems to obtain sufficient numbers of reliable slip models, which are required for a statistical discussion.

\section{References}

Bouchon, M., Toksöz, N.M., Karabulut, H., Bouin, M.-P., Dietrich, M., Aktar, M., and Edie, M., 2002. Space and time evolution of rupture and faulting during the 1999 Izmit (Turkey) earthquake. Bull. Seismol. Soc. Am., 92:256-266, doi:10.1785/0120000845.

Delouis, B., Giardini, D., Lundgren, P., and Salichon, J., 2002. Joint inverstion of InSAR, GPS, teleseismic, and strong motion data for the spatial and temporal distribution of earthquake slip: Application to the 1999 Izmit mainshock. Bull. Seismol. Soc. Am., 92:278-299, doi:10.1785/0120000806.

Ide, S. and Aochi, H., 2005. Earthquakes as multiscale dynamic ruptures with heterogeneous fracture surface energy. $J$. Geophys. Res., 110:B11303, doi:10.1029/2004JB003591.
Ide, S., Beroza, G.C., and McGuire, J.J., 2005. Imaging earthquake source complexity. Seismic Earth: Array Analysis of Broadband Seismograms. Geophys. Monogr. Ser. 157:117135.

Mai, P.M. and Beroza, G.C., 2000. Source scaling properties from finite-fault-rupture models. Bull. Seismol. Soc. Am., 90:604614, doi:10.1785/0119990126.

Sekiguchi, H. and Iwata, T., 2002. Rupture process of the 1999 Kocaeli, Turkey, earthquake estimated form strong-motion waveforms. Bull. Seismol. Soc. Am., 92:300-311, doi:10.1785/0120000811.

Somerville, P., Irikura, K., Graves, R., Sawada, S., Wald, D., Abrahamson, N., Iwasaki, Y., Kagawa, T., Smith, N., and Kowada, A., 1999. Characterizing crustal earthquake slip models for the prediction of strong ground motion. Seismol. Res. Lett., 70:59-80.

Uchide, T. and Ide, S., 2006. Development of multiscale slip inversion method and its application to the 2004 mid-Niigata prefecture earthquake. Eos Trans. AGU, 87(52), Fall Meet. Suppl., Abstract S42C-01.

Yagi, Y. and Kikuchi, M., 2000. Source rupture process of the Kocaeli, Turkey, earthquake of August 17, 1000, obtained by joint inversion of near-field data and teleseismic data. Geophys. Res. Lett., 27:13-1969.

Yamada, T., Mori, J.J., Ide, S., Kawakata, H., Iio, Y., and Ogasawara, H., 2005. Radiation efficiency and apparent stress of small earthquakes in a South African gold mine. J. Geophys. Res., 110:B01305, doi:10.1029/2004JB003221.

\section{Author}

Satoshi Ide, Department of Earth and Planetary Science, University of Tokyo, 7-3-1, Hongo, Bunkyo, Tokyo, 113-0033, Japan, e-mail: ide@eps.s.u-tokyo.ac.jp 